\title{
Antioxidant properties of resveratrol and piceid on lipid peroxidation in micelles and monolamellar liposomes
}

\author{
Sabrina Fabris ${ }^{a}$, Federico Momo ${ }^{\mathrm{a}, *}$, Giampietro Ravagnan $^{\mathrm{b}}$, Roberto Stevanato ${ }^{\mathrm{a}}$ \\ a Department of Physical Chemistry, University of Venice, Dorsoduro, 2137, 30123 Venezia, Italy \\ b Department of Environmental Sciences, University of Venice, Dorsoduro, 2137, 30123 Venezia, Italy
}

\section{A R T I C L E I N F O}

Article history:

Received 12 February 2008

Received in revised form 19 March 2008

Accepted 19 March 2008

Available online 29 March 2008

\section{Keywords:}

Piceid

Resveratrol

Antioxidants

Lipid peroxidation

Phospholipid membranes

\begin{abstract}
A B S T R A C T
The antioxidant activities of trans-resveratrol (trans-3,5,4'-trihydroxystilbene) and trans-piceid (trans-5,4'dihydroxystilbene-3-0- $\beta$-D-glucopyranoside), its more widespread glycosilate derivative, have been compared measuring their inhibitory action on peroxidation of linoleic acid (LA) and the radical scavenging ability towards different free radicals (such as DPPH) and radical initiators. It has been found that the two stilbenes have similar antioxidant capacity, while the comparison with BHT (2,6-di-tert-butyl-4-methylphenol) and $\alpha$-tocopherol (vitamin E, vit. E), taken as reference, points out a slower but prolonged protective action against lipid peroxidation. Furthermore, piceid appears more efficacious than resveratrol as a consequence of the reaction of the latter with its radical form.

The DSC profiles of phosphatidylcholine liposomes of various chain lengths, and EPR measurements of spin labelled liposomes demonstrated that the susceptible hydroxyl group of these compounds are located in the lipid region of the bilayer close to the double bonds of polyunsatured fatty acids, making these stilbenes particularly suitable for the prevention and control of the lipid peroxidation of the membranes.
\end{abstract}

(c) 2008 Elsevier B.V. All rights reserved.

\section{Introduction}

It is known that a diet rich in fruit and vegetables helps to prevent cardiovascular diseases, cancer, and ageing-related disorders [1].

The effect can be traced back to the antioxidant activity of polyphenols of fruits and vegetables, able to inhibit lipid peroxidation, forced by free radicals, and related to chronic health problems like cancer, arthrosclerosis and ageing [2-4]. In particular resveratrol, a polyphenol mainly present in grapes and red wine, demonstrated interesting biomedical properties for its inhibitory effects on cancer promotion and propagation [5], cardioprotective action due to inhibition of the oxidation of low-density lipoprotein (LDL) [6] and of platelet aggregation [7,8], anti-inflammatory activities and preventive effect on Alzheimer's disease and dementia [9].

Recently it was found that, in the grape juices, the average concentration of piceid, the glycoside form of resveratrol, is seven times that of resveratrol [10] and this is probably the most abundant form of resveratrol in nature [11].

A number of studies hypothesise that piceid may have biomedical properties similar to those above mentioned for resveratrol: anticarcinogenic effects [12,13] and inhibition of platelet aggregation $[14,15]$ and of oxidation of LDL [16].

\footnotetext{
* Corresponding author. Tel.: +39 041 2348599; fax: +39 0412348594. E-mail address: momo@unive.it (F. Momo).
}

The aim of this work is to understand if, by analogy, piceid has also antioxidant properties comparable to resveratrol.

Unfortunately the antioxidant capacity of a compound and its effectiveness in preventing diseases cannot be unequivocally determined. Roughly the experimental methods can be grouped into two classes, based on hydrogen atom transfer (HAT) and on single electron transfer (ET) [17]. Therefore, we studied the antioxidant activity of resveratrol and piceid in terms of their inhibitory action on peroxidation of linoleic acid (LA), an HAT assay, and as radical scavenging ability towards 2,2'-diphenyl-1-picrylhydrazyl (DPPH method), an ET assay. The scavenging reaction of resveratrol is accompanied by the formation of a product which is not formed in the analogue reaction of piceid, and the data support the hypothesis of a reaction between one molecule of resveratrol and one molecule of its radical. This point, the possibility that an antioxidant may react with itself, is of general interest and must be accounted for in evaluating the antioxidant activity of a molecule.

At membrane level there is an assumed relationship between the rate of lipid peroxidation and membrane composition and fluidity [18-20], implying that the efficacy of an antioxidant can be meaningfully modulated by its collocation inside the bilayer. From this point of view, liposomes are much more representative models of biological membranes than micelles, so the peroxidation measurements were repeated in monolamellar liposomes. Contemporarily the interactions of resveratrol and piceid with multilamellar liposomes of satured L- $\alpha$ phosphatidylcholine (PC) of various chain lengths (dimyristoyl, DMPC, dipalmitoyl, DPPC, distearoyl, DSPC) were investigated, determining 
<smiles>Oc1ccc(/C=C/c2cc(O)cc(O)c2)cc1</smiles>

trans-3,5,4'-trihydroxystilbene

(trans-Resveratrol)<smiles>Cc1cc(C(C)(C)C)c(O)c(C(C)(C)C)c1</smiles>

2,6-di-tert-butyl-4-methylphenol (BHT)<smiles>OCC(O)C(O)OC(Oc1cc(O)cc(C=Cc2ccc(O)cc2)c1)C(O)CO</smiles>

trans-5,4'-dihydroxystilbene3-O- $\beta$-D-glucopyranoside

(trans-Piceid)<smiles>C=C(C)CCCC1(C)CCc2c(C)c(O)c(C)c(C)c2O1</smiles>

$$
\alpha \text {-Tochopherol }
$$

(vit. E)

Fig. 1. Molecular structure of studied compounds.

the partition coefficients of the drugs, the decay of the ordered lipid organisation they produce, and their position in membranes using differential scanning calorimetry (DSC) and spin labelling EPR techniques.

Data point out how both these compounds are located in the lipid region of bilayer close to double bounds of LA and have, on average, a slower but longer antioxidant capacity than BHT and $\alpha$-tocopherol taken as reference (Fig. 1).

The comparison between micelles and monolamellar liposomes demonstrated as well that the inhibitory effect of vit. E, measured in liposomes, is about one half the same effect when measured in micelles. This will be explained in terms of a different accessibility of the phenolic groups of vit. E to the double bonds of unsaturated lipid chains, and demonstrated that the true antioxidant power of vit. $\mathrm{E}$ could be overestimated by usual measurements in micelles.

\section{Materials and methods}

\subsection{Chemicals}

All chemicals, of the highest available quality, were obtained from Sigma Chemical Co. (St. Louis, USA). ABIP was a kind gift of Wako Chemicals USA. Trans-piceid and trans-resveratrol, with a purity grade higher than $99 \%$, have been supplied by the Istituto Agrario di S. Michele all'Adige (IT) [21]. The aqueous solutions were prepared with quality milliQ water.

\subsection{Inhibition of lipid peroxidation}

The antioxidant activity of resveratrol and piceid to prevent LA peroxidation was studied in sodium dodecyl sulfate (SDS) micelles and in monolamellar liposomes of DPPC.

The peroxidation was initiated by the thermolabile water-soluble azo initiator 2,2'-azobis[2-(2-imidazolin-2-yl)propane] (ABIP) and measured as rate of oxygen consumption. The reaction was carried on in a closed thermostated vessel $\left(37^{\circ} \mathrm{C}\right)$ and the oxygen consumption was followed using a Clark electrode.

In SDS micelles ( $50 \mathrm{mM}$ ) the concentration of LA was $10 \mathrm{mM}$, while in DPPC monolamellar liposomes ( $20 \mathrm{mM}$ ) LA was $2.5 \mathrm{mM}$; the choice of the concentrations was the result of a series of tests at different concentrations and represents the best compromise between a high rate of peroxidation and a minor change in lipid organization due to LA [22]; in both cases we used phosphate buffer (50 mM, pH 7.4).

To the solution, previously equilibrated with air and kept at constant temperature $\left(3{ }^{\circ} \mathrm{C}\right)$, the azo initiator ABIP ( $4 \mathrm{mM}$ final concentration) was injected; and the oxygen consumption was monitored. After a few minutes an ethanol solution of antioxidant was added $\left(2 \cdot 10^{-6} \mathrm{M}\right.$ final concentration). Every experiment was repeated three times.

The antioxidant capacity was calculated as change of slope of oxygen consumption before and after the addition of the antioxidant; in particular it is expressed by the percentage inhibition of the peroxidation [23]:

$P_{\text {inh }} \%=\frac{R_{\mathrm{p}}-R_{\text {inh }}}{R_{\mathrm{p}}} \cdot 100$

Where $R_{\mathrm{p}}$ is the rate of propagation of lipid peroxidation due to $\mathrm{ABIP}$ and $R_{\mathrm{inh}}$ is the rate of inhibition of peroxidation after the addition of antioxidant.

\subsection{2,2-diphenyl-1-picrylhydrazyl (DPPH) radical scavenging capacity assay}

This method is based on the capacity of an antioxidant to scavenge the stable free radical DPPH [24]. In the reaction DPPH goes through a change of color so its disappearance can be monitored spectrophotometrically at $515 \mathrm{~nm}\left(\varepsilon=10,800 \mathrm{M}^{-1} \mathrm{~cm}^{-1}\right)$ [25]. The reaction was carried on at $25{ }^{\circ} \mathrm{C}$ in ethanol at various antioxidant concentrations $(12.5,25,50 \mu \mathrm{M})$, while that of DPPH was fixed at $100 \mu \mathrm{M}$. 


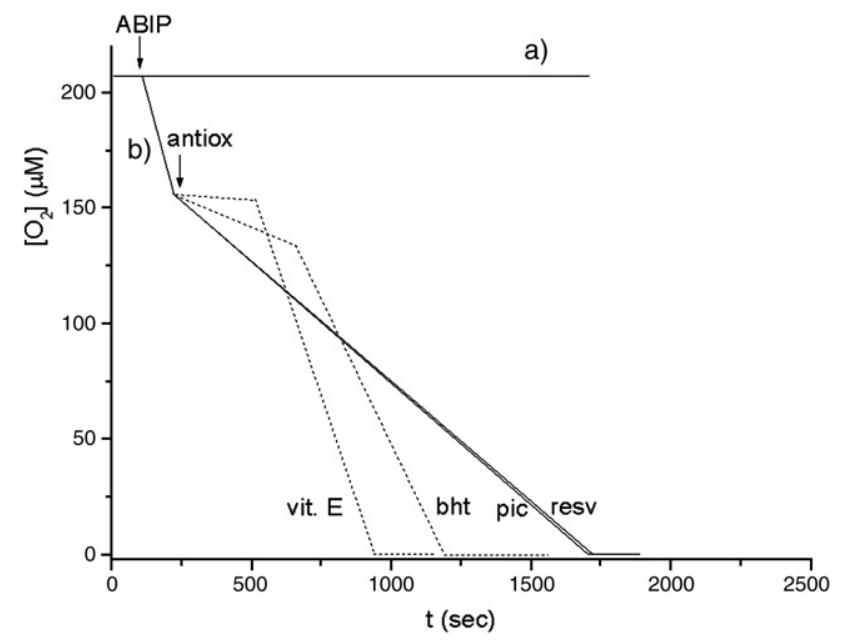

Fig. 2. Oxygen consumption during ABIP-initiated peroxidation of LA ( $10 \mathrm{mM})$ in SDS micelles (50 mM): a) in the absence of ABIP, b) after addition of ABIP (4 mM), and in the presence of antioxidant $(2 \mu \mathrm{M}) ; \mathrm{pH} 7.4, T=37^{\circ} \mathrm{C}$.

The radical scavenging ability towards DPPH was calculated as decrease of DPPH concentration in the first $40 \mathrm{~s}$ of reaction and expressed as initial reaction rate $\left(V_{0}\right)$.

\subsection{Reaction between radical initiators and antioxidants}

Our compounds absorb in the UV region, so it is possible to follow spectrophotometrically their reaction with ABIP measuring their disappearance in the time.

Resveratrol and piceid show the same molar absorptivity, that is $\varepsilon_{317}=30,000 \mathrm{M}^{-1} \mathrm{~cm}^{-1}$, while for BHT is $\varepsilon_{272}=1100 \mathrm{M}^{-1} \mathrm{~cm}^{-1}$.

The buffer solution and the reaction temperature conditions were the same of lipid peroxidation and the initial rate is calculated in the first 5 min of reaction.

The very high hydrophobicity of vit. E made the determination of its reactivity with ABIP impossible in water solution.

\subsection{Liposome preparation}

Multilamellar vesicles were prepared following the method of Kusumi et al. [26]. Phospholipids were dissolved in a 2:1 chloroform methanol mixture then dried with a stream of nitrogen gas and kept under vacuum for at least $14 \mathrm{~h}$. The dried lipids were suspended, when not otherwise specified, in a HEPES $0.1 \mathrm{M}, \mathrm{pH} 7.2$, buffer. The lipid dispersion, with a $101 \mathrm{mM}$ final lipid concentration, was warmed over the transition temperature, mixed vigorously with a vortex for $30 \mathrm{~s}$ five times and used just as obtained for DSC measurements. When required, spin labels (1.8 $\mathrm{mM}$ final concentration) were added to the chloroform methanol phospholipid mixture.

Unilamellar liposomes were prepared combining the freezethawing and extrusion protocol [27], starting from a $500 \mu \mathrm{l}, 40 \mathrm{mM}$, phospholipid suspension. Extrusion was performed using the Avestin Liposofast apparatus, with $100 \mathrm{~nm}$ pore size filters.

\subsection{Determination of the partition coefficients}

The liposome-water partition coefficients of resveratrol and piceid were experimentally determined as described in Ref. [28].

\subsection{UV-VIS, DSC and EPR measurements}

Spectrophotometric measurements were recorded on a UV-VIS Beckman DU 640 instrument equipped with a thermostated quartz cell.
Calorimetric experiments were performed on a Setaram DSC 92. About $50 \mathrm{mg}$ of phospholipid dispersion was placed in an aluminium crucible, while an identical crucible was filled with an equivalent weight of HEPES solution and placed in the reference cell. The temperature scanning rate was $0.5{ }^{\circ} \mathrm{C} \mathrm{min}^{-1}$. The transition temperature $T_{\mathrm{C}}$ from the gel-to-fluid phase of liposomes was taken at the peak of the DSC profiles.

EPR measurements were performed on a Bruker ER 200 D, $9 \mathrm{GHz}$ spectrometer. Samples were placed in a gas permeable TPX tube $1 \mathrm{~mm}$ i.d. (Wilmad, N.J. USA) and centered in the resonant cavity, then deoxygenated under nitrogen flow above $T_{\mathrm{c}}$ for $10 \mathrm{~min}$.

\section{Results and discussion}

\subsection{Antioxidant activity}

\subsubsection{Inhibition of lipid peroxidation}

Fig. 2 shows the oxygen consumption vs time of the ABIP-initiated lipid peroxidation in LA/SDS micelles. The oxygen concentration stays unchanged till the addition of ABIP: when ABIP is added, the oxygen concentration decreases with a rate of $4 \cdot 10^{-7} \mathrm{M} \mathrm{s}^{-1}$; this rate is differently slowed down by the antioxidants: vit. E and BHT are able to stop almost completely the peroxidation reaction for an inhibition period $\left(t_{\text {inh }}\right)$ of a few minutes, during which the consumption of oxygen is negligible; then the peroxidation restarts with roughly the same original rate of propagation of ABIP, as a consequence of the loss of the antioxidant capacity of the compounds. On the contrary, piceid and resveratrol do not display the inhibition time and their rates of oxygen consumption are almost constant and slower than those of BHT and vit. E after $t_{\text {inh. }}$. From this point of view, piceid and resveratrol could be considered less reactive antioxidants than BHT and vit. E, but having a prolonged protective effect against radicals.

The lipid peroxidation was studied in liposomial system too (Fig. 3), as monolamellar liposomes are structurally more similar to cell membranes than micelles. In this case a lower rate of propagation was observed $\left(4 \cdot 10^{-8} \mathrm{M} \mathrm{s}^{-1}\right)$, which can be due both to the different concentration of LA in the two systems and to a different permeability of ABIP in the two matrices; the general trend of peroxidation inhibition is similar to that in micelles, with the exception of vit. $\mathrm{E}$ which, when inserted in a phospholipid bilayer, does not display any inhibition period and loses one half of its antioxidant power (Fig. 4) The observation may be of some relevance because it demonstrates that the choice of a lipid moiety, micelles, rather than liposomes, can distort the scale of effectiveness of the antioxidants. Moreover, as it

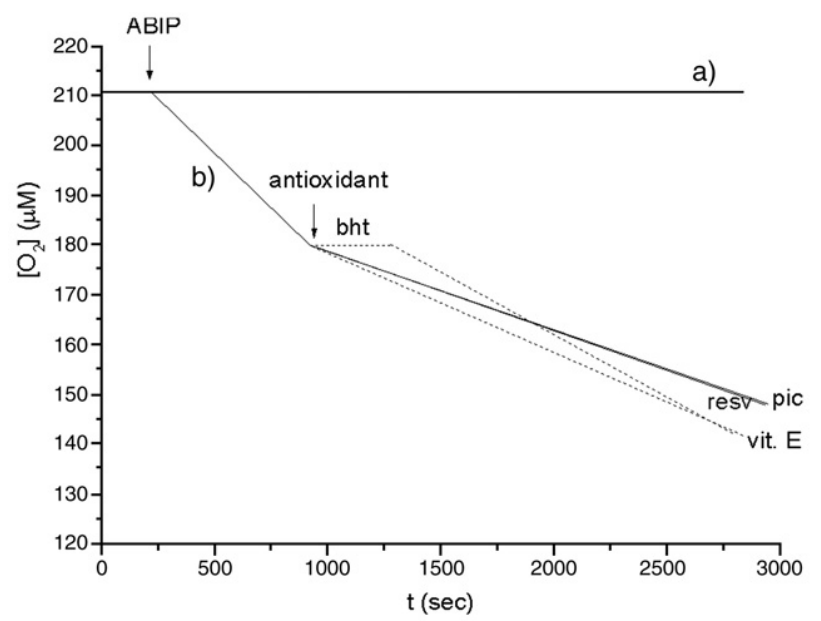

Fig. 3. Oxygen consumption during ABIP-initiated peroxidation of LA ( $2.5 \mathrm{mM})$ in monolamellar DPPC liposomes (20 mM): a) in the absence of ABIP, b) after addition of $\mathrm{ABIP}(4 \mathrm{mM})$, and in the presence of antioxidant $(2 \mu \mathrm{M}) ; \mathrm{pH} 7.4, T=37{ }^{\circ} \mathrm{C}$. 


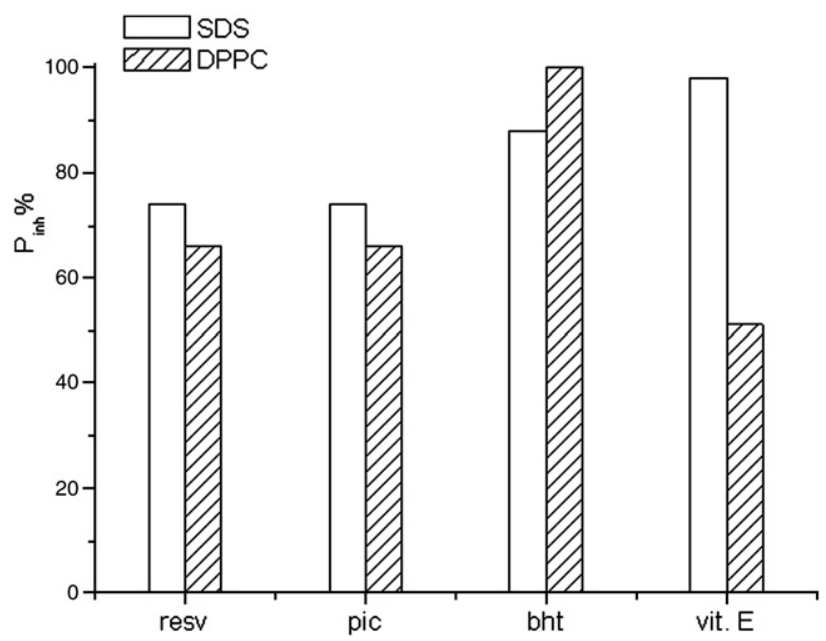

Fig. 4. Percentage inhibition $\left(P_{\text {inh }} \%\right)$ of the ABIP-initiated peroxidation of LA in SDS micelles and DPPC monolamellar liposomes after addition of antioxidants $(2 \mu \mathrm{M}) ; \mathrm{pH}$ $7.4, T=37^{\circ} \mathrm{C}$. For BHT and Vit. E the percentage is calculated during the inhibition period.

comes up from EPR studies, these molecules have different positions inside the bilayer, and this is a further modulating factor of their inhibitory action.

\subsubsection{Radical scavenging capacity toward $D P P H$}

It is commonly accepted that the first reaction step between an antioxidant and DPPH is the abstraction of a hydrogen atom from the antioxidant to give diphenylpicrylhydrazine (DPPH-H) and a phenoxyl radical [29] which will undergo further reactions such as dimerization, complexation and donation of a second hydrogen atom [30].

The initial rates $V_{0}$ of the reaction between $\mathrm{DPPH}$ and the antioxidants, as a function of the antioxidant concentration, are reported in Fig. 5. At $50 \mu \mathrm{M}$ concentration, vit. E shows a scavenging effect about 5 times faster than the other compounds; resveratrol and piceid, from this point of view, are not so efficient, but still remain comparable to BHT.

\subsubsection{Reaction between radical initiators and antioxidants}

Literature reports that antioxidant capacity of polyphenols is due to their ability to stop or to slow down lipid peroxidation, reacting with peroxyl radicals forming during the propagation phase [31,32]. Anyway, because by definition an antioxidant is a molecule able to react with all radicals present in the solution, we studied the reactivity of resveratrol and piceid toward ABIP, as the possibility exists that the

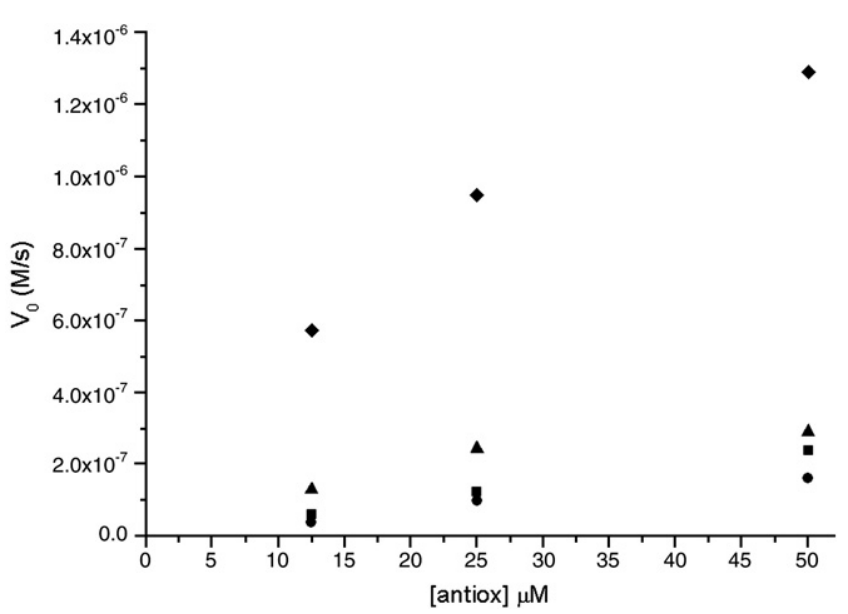

Fig. 5. Initial reaction rate $\left(V_{\mathrm{o}}\right)$ of DPPH $(100 \mu \mathrm{M})$ disappearance vs. antioxidant concentration at $515 \mathrm{~nm}$ and $25{ }^{\circ} \mathrm{C}$ in ethanol. vit. E; $\boldsymbol{\Delta}$ BHT; $\boldsymbol{\square}$ resv; pic.

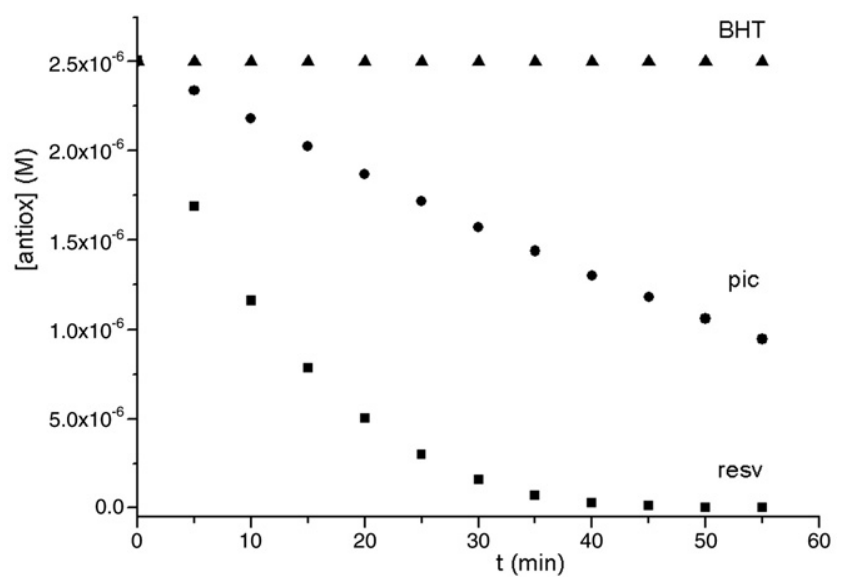

Fig. 6. Decay of antioxidant concentration for the reaction with ABIP vs. time at $37{ }^{\circ} \mathrm{C}$ and $\mathrm{pH}$ 7.4. [antioxidant $]=2.5 \cdot 10^{-5} \mathrm{M},[\mathrm{ABIP}]=2 \cdot 10^{-3} \mathrm{M}$.

lipid peroxidation is inhibited at the beginning by a direct interaction between antioxidants and radical initiators.

The consumption of antioxidant during the reaction with ABIP is plotted in Fig. 6: resveratrol and piceid concentrations decrease with the time, while that of BHT stays constant, meaning that resveratrol and piceid, unlike BHT, react with ABIP.

At our experimental conditions, the kinetic rate constant of ABIP decomposition is $1.9 \cdot 10^{-4} \mathrm{~min}^{-1}$ [33] and its rate of decomposition is $3.3 \cdot 10^{-7} \mathrm{M} \mathrm{min}^{-1}$, which is almost identical to the value of $3.2 \cdot 10^{-7} \mathrm{M}^{-1}$ $\mathrm{min}^{-1}$ we found for the initial rate of consumption of piceid, while that of resveratrol is $1.6 \cdot 10^{-6} \mathrm{M}^{-1} \mathrm{~min}^{-1}$, that is five times bigger.

This means that the rate of reaction between piceid and ABIP is limited by the thermolysis of ABIP and the production of carbon-centered amidinium radical. The higher rate of consumption of resveratrol implies secondary reactions between one resveratrol molecule and its radical form, generated from the reaction with ABIP, which can lead to the formation of new compounds, like dimmers, quinones and polymer. The formation of a secondary product is confirmed by the UV-VIS spectra which display two new bands with a maximum absorbance at 265 and $360 \mathrm{~nm}$ (Fig. 7a), not present in the case of piceid (Fig. 7b).

The reactivity of resveratrol and piceid towards initiators or stable free radicals, with trends similar to those reported in Fig. 6, is confirmed also with AAPH (2,2'-azobis(2-amidinopropane)) and ABCPA (4,4'azobis(4-cyanopentanoic acid)), with the stable spin label TEMPO (2,2,6,6-tetramethyl-1-piperidinyloxy), with hydrogen peroxide and with the couple hydrogen peroxide-horseradish
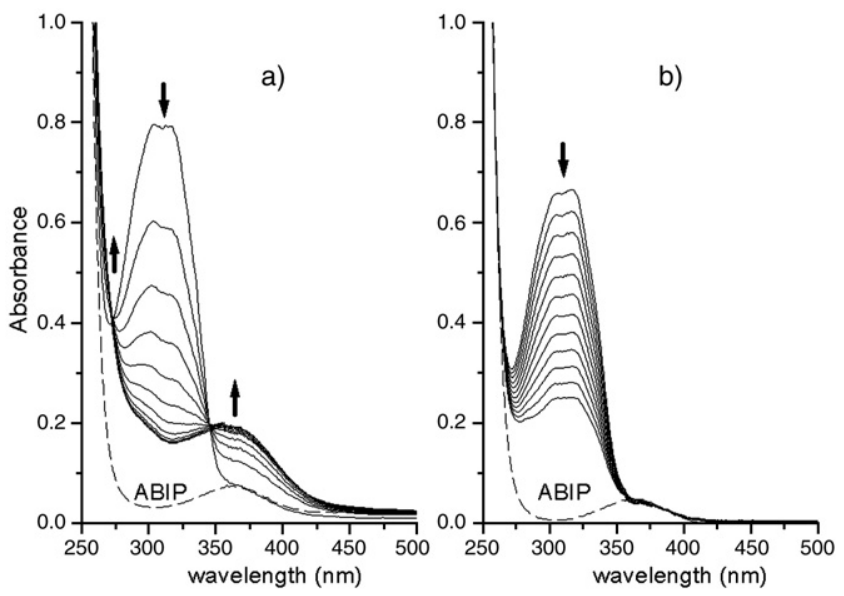

Fig. 7. Spectrophotometric recording of the reaction between: a) resveratrol, b) piceid and $\mathrm{ABIP}$ at $37^{\circ} \mathrm{C}$ and $\mathrm{pH} 7.4$. The elapsed time between scans was 5 min [antioxidant] $=$ $2.5 \cdot 10^{-5} \mathrm{M},[\mathrm{ABIP}]=2 \cdot 10^{-3} \mathrm{M}$. 


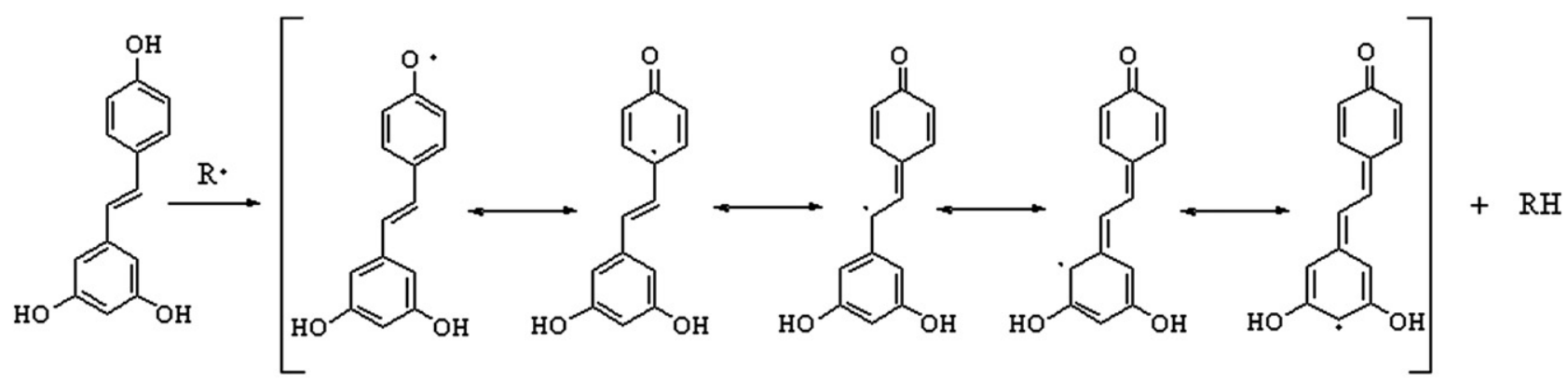

(A)

quinone methide

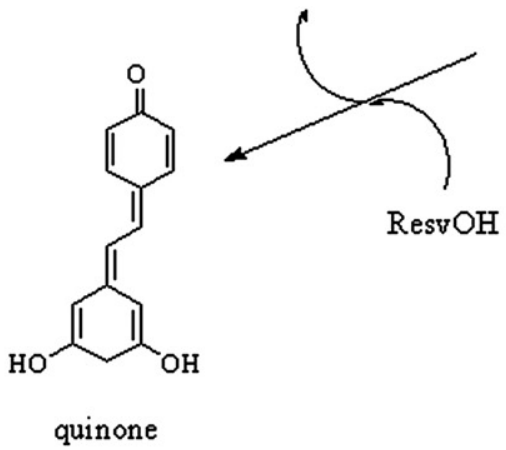

Fig. 8. Possible mechanism of reaction between resveratrol and a radical initiator.

peroxidase (HRP), (data not shown). In all cases, BHT does not react, while we observe a faster decrease of resveratrol concentration with respect to piceid.

In literature it is generally accepted that the reaction between resveratrol and HRP-hydrogen peroxide leads to the formation of a resveratrol dehydrodimer known like $\delta$-vinifrin [34] which can be hardly identified with the secondary product we found. In fact, $\delta$ vinifrin has an UV spectrum similar to resveratrol, centered at $307 \mathrm{~nm}$ [35] and does not show the two bands at about 275 and $360 \mathrm{~nm}$.

Thompson [36] demonstrated that the oxidation of eugenol by hydrogen peroxide in the presence of horseradish peroxidase (HRP) leads to the formation of a quinone methide which absorbs at 270 and $350 \mathrm{~nm}$. We hypothesize, by analogy, that, also in our case, a quinone form of resveratrol is formed by the oxidative reaction (Fig. 8). The discriminating factor between the different reaction pathways, leading to the quinone or to the dimer forms, is most likely the initial concentration of radical resveratrol: in fact the dimerization involves two radical molecules and is, by consequence, favored by a high concentration of radical resveratrol.

The question is now to understand the different behaviour between resveratrol and piceid, that differ only for a glycoside group in place of a hydroxyl group. Resveratrol has a phenol moiety, due to a hydroxyl group in $4^{\prime}$-position and a m-hydroquinone moiety due to hydroxyl groups in 3,5-positions (Fig. 1). It has been demonstrated that the 4'-hydroxyl is the most reactive in scavenging free radicals because the corresponding phenoxly radical can delocalize the unpaired electron on the whole molecule [37,38], and it is possible

Table 1

Values of $\log P$ and percent fraction (\%) of antioxidant bound to liposomes in the gel state for the various systems; DMPC (C14:0), DPPC (C16:0), DSPC (C18:0)

\begin{tabular}{lllll}
\hline & \multirow{2}{*}{ Octanol-water } & \multicolumn{3}{l}{ Liposomes-water } \\
\cline { 3 - 5 } & & DMPC (\%) & DPPC (\%) & DSPC (\%) \\
\hline Resveratrol & 1.87 & $2.63(93)$ & $3.07(97)$ & $3.11(98)$ \\
Piceid & 0.89 & $2.43(89)$ & $2.27(86)$ & $2.57(93)$ \\
\hline
\end{tabular}

that the most stable resonance form of the resveratrol radical is that where the unpaired electron is located in the 4-position close to 3 and 5 hydroxyl groups (Fig. 8, form A). The lack of formation of a dimer of
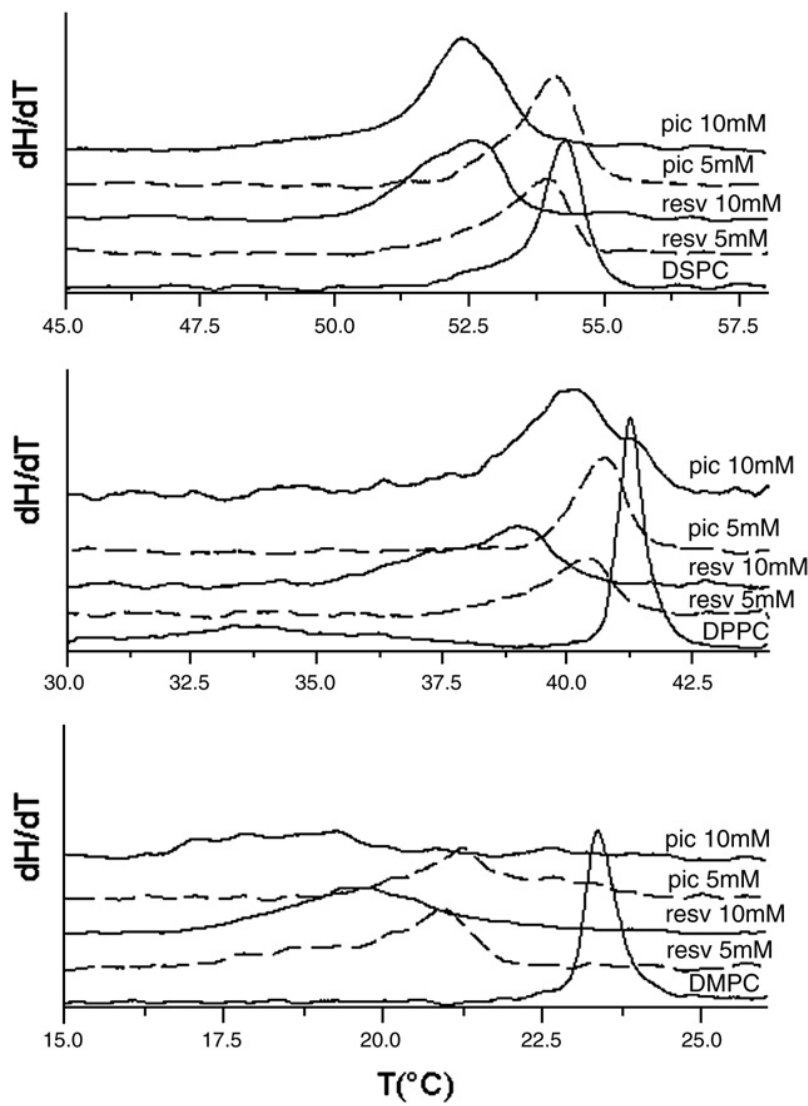

Fig. 9. DSC profiles of the gel-to-fluid state transition of PC multilamellar liposomes $(100 \mathrm{mM})$ doped with resveratrol and piceid $(5$ and $10 \mathrm{mM}) . y$ axis: $\mathrm{dH} / \mathrm{dT}$ in arbitrary units; $x$ axis: temperature $T$ in ${ }^{\circ} \mathrm{C}$. 


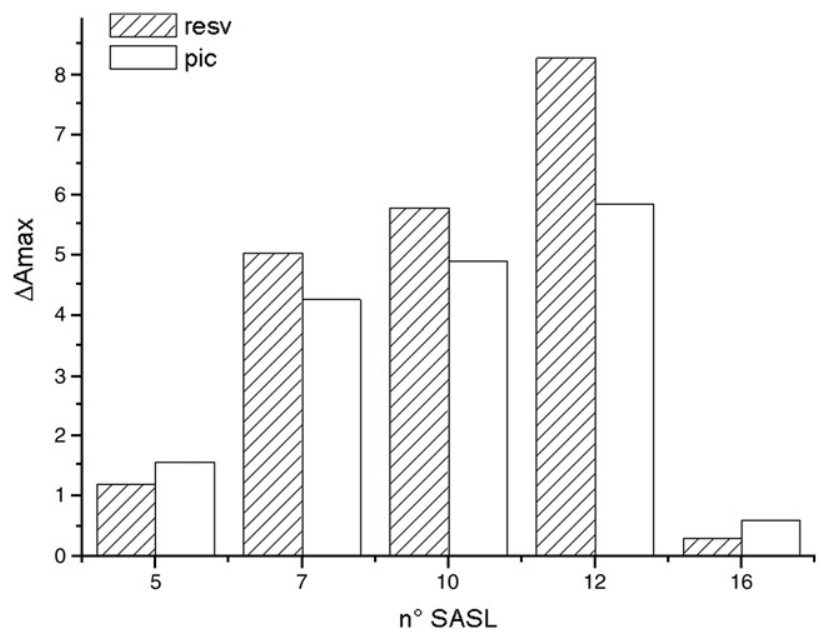

Fig. 10. The differences $\left(\Delta A_{\max }\right)$ between the $A_{\max }$ values (in $10^{-4}$ Tesla) with and without antioxidant in DPPC liposomes below the transition temperature vs. spin label position $(n)$. DPPC $100 \mathrm{mM}$, piceid and resveratrol $10 \mathrm{mM}$.

piceid could be explained on the same basis, as the substitution of a hydroxyl for a glycoside group makes the radical more stable for steric hindrance and prevents the reaction with another molecule of piceid.

\subsection{Interactions of antioxidants with membranes}

\subsubsection{Partition coefficients}

The logarithm of octanol-water partition coefficient $(\log P)$ is used as a measure of the hydrophobicity of compounds and it is assumed to represent the general tendency of a chemical to partition between an aqueous and an organic phase. $P$ is defined as $P=\left(n_{\text {oct }} / V_{\text {oct }}\right) /\left(n_{\mathrm{w}} / V_{\mathrm{w}}\right)$, where $n$ denotes the number of moles of antioxidant, $V$ the volume, and ${ }_{\text {oct }}$ and ${ }_{\mathrm{w}}$ refer to octanol and water phase respectively.

It must be said that the notion of partition coefficient, when extended to liposomes/water dispersions, can be vague for a number of reasons, not least the difficulty of defining the volume of liposomes and considering it like a homogeneous phase; anyway it is commonly accepted and $\log P$ is used to quote the fraction of dopant adsorbed into the membrane.
The values of $\log P$ and the percent fraction (\%) of antioxidant bound to liposomes in the gel state, are given in Table 1 .

Data demonstrate that, as expected, the hydrophobicity is dependent on the substitution of the $m$-hydroxy group by a glucose residue, that makes piceid less hydrophobic than resveratrol; in any case both of them are quite lipophylic molecules, which, in a water dispersion of multilamellar liposomes are almost exclusively present in the lipid phase.

\subsubsection{DSC studies}

In Fig. 9 the DSC profiles of the gel-to-fluid state transition of DMPC (C14:0), DPPC (C16:0), DSPC (C18:0) multilamellar liposomes at increasing concentration of resveratrol and piceid are plotted.

Data show that the effects of resveratrol and piceid are similar: at increasing antioxidants concentration the DSC profiles are broadened and shifted towards lower temperatures; these effects are particularly evident in the case of piceid in DMPC liposomes where at $10 \mathrm{mM}$ of the stilbene the transition is hardly detectable.

Because there is no reason to think that the interaction of resveratrol and piceid with DMPC is not the same as that of DPPC and DSPC, it is likely that the different behaviour in DMPC liposomes is related to the length of acyl chains: the longer are the acyl chains, the more stable and the less perturbable is the bilayer.

The spreading of the peaks suggests that the presence of the antioxidants induces the formation of defects in the ordered structure of membrane which is reflected in the fall of cooperativity; the decrease of transition temperature is a sign of a much looser bilayer. The contemporary featuring of these trends is usually determined by dopants localized in the interface region of bilayer.

\subsubsection{EPR measurements}

EPR spectra have been collected for stearic acids, spin labeled at the 5 th, 7th, 10th, 12th and 16th carbon positions ( $n$-SASL) and incorporated in liposomes of DPPC, with and without dopants, as described in liposomes preparation.

The spectra were recorded below and above the gel-to-fluid transition temperature of DPPC with analogous results. Here we reported the values obtained in the gel phase and expressed as $A_{\max }$ (the separation between the outer hyperfine lines) which is the most appropriate parameter for monitoring the effects of dopants in the gel phase [39]. The differences between the $A_{\max }$ values with and without antioxidant are reported in Fig. 10.

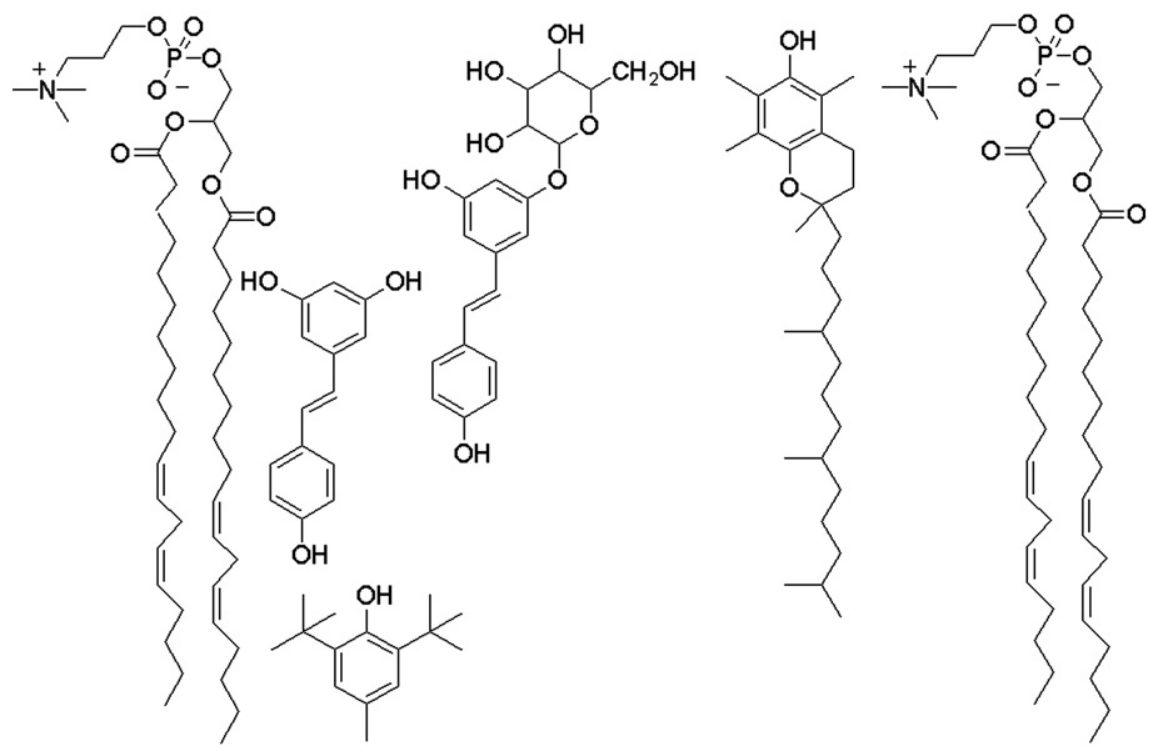

Fig. 11. Possible collocation of dopants in the membrane. 
As it emerges from the graph, the more marked variations of $A_{\max }$ are related with the 7th, 10th and 12th carbon positions for both the molecules, making plausible that the stilbenes compounds are located in this zone of bilayer, in some way anchored to the interfacial or polar region, with piceid closer to the membrane surface according to its higher hydrophilicity. As a consequence, this positioning facilitates the antioxidant action of the two molecules as the reactive hydroxy groups of resveratrol and piceid fall very near to the double bonds of polyunsatured fatty acid chains (PUFA) (Fig. 11). Similar arguments are valid for BHT as well, which is a high lipophilic molecule, most likely located in the deepest region of the bilayer as can be gathered by analogy with 2,6-ditertbutylphenol [40].

Instead the hydroxy group of vit. $E$ is close to the polar head groups of the phospholipids [41]; thus in the presence of lipid peroxidation, resveratrol, piceid and BHT can react more quickly with the peroxyl radical than vit. E for distance motives. It has been hypothesized [42] that, once lipid peroxyl is formed, it diffuses to the surface of the membrane and in this way can be trapped by the hydroxy group of vit. $\mathrm{E}$. If we suppose that this floating is faster in micelles than in the bilayer, we can explain the different activity of vit. $\mathrm{E}$ in liposomes and in micelles.

\section{Conclusions}

A univocal classification of the antioxidant substances is not easy, given the manifold types of reactions which are involved, and, actually, the antioxidant power coincides with the methods we use for determining it. Multiple tests, representative of various radical mechanisms, are perhaps the best strategy for stating an empirical and approximate scale of effectiveness.

In this paper resveratrol and piceid were compared with two common antioxidants like vit. E and BHT, in terms of inhibitory effect on lipid peroxidation and radical scavenging ability.

From this point of view, the following considerations can be underlined:

i) resveratrol and piceid show similar behaviour as inhibitors of the lipid peroxidation; maybe piceid and resveratrol are, on average, slightly less efficacious than vit. E and BHT, but their slower reactivity against lipid peroxidation combined to a prolonged protective action, could be of interest in clinical or dietary applications;

ii) a greater reactivity with different types of radicals or initiators has been found for the stilbenes with respect to BHT, suggesting a wide action spectrum against radical injury, i.e. a potential preventive effect;

iii) the lipophilicity of resveratrol and piceid and their collocation into the membrane with the susceptible hydroxyl group close to the double bonds of PUFA, make these stilbenes particularly suitable for the prevention and control of the lipid peroxidation in membranes;

iv) as demonstrated in the case of resveratrol, the possibility that an antioxidant may react with itself must be accounted for in evaluating the antioxidant activity of a molecule, because it can result in wasting precious antioxidant agent. From this point of view, piceid appears more efficacious than resveratrol;

As a side remark, we want to stress the striking difference of vit. E antioxidant power observed in micelles and monolamellar liposomes: a great care must be taken when a biological value has to be assigned to data from micelles, which are by far the most common lipid moiety in lipid peroxidation experiments, but not the best model of biological membrane.

\section{Acknowledgement}

The authors thank Fulvio Mattivi of the Istituto Agrario di S. Michele all'Adige (IT) for the supply of piceid and resveratrol.

\section{References}

[1] W.C. Willet, Eat, Drink, and Be Healthy: The Harvard Medical School Guide to Healthy Eating, McGraw-Hill, New York, 2001.

[2] L. Marnett, Oxyradicals and DNA damage, Carcinogenesis 21 (2000) 361-370.

[3] B. Halliwell, J.M.C. Gutteridge, Free Radicals in Biology and Medicine, 3rd ed.Oxford University Press, Oxford, 1999.

[4] H. Esterbauer, P. Ramos, Chemistry and pathophysiology of oxidation of LDL, Rev. Physiol., Biochem. Pharmacol. 127 (1996) 31-64.

[5] L. Fremont, Biological effects of resveratrol, Life Sci. 66 (2000) 663-673.

[6] E.N. Frankel, A.L. Waterhouse, J.E. Kinsella, Inhibition of human LDL oxidation by resveratrol, Lancet 24 (1993) 1103-1104.

[7] A.A. Bertelli, L. Giovannini, D. Riannessi, M. Migliori, W. Bernini, M. Fregoni, A Bertelli, Antiplatelet activity of synthetic and natural resveratrol in red wine, Int. J. Tissue React. 17 (1995) 1-3.

[8] C.R. Pace-Asciak, S. Hahn, E.P. Diamandis, G. Soleas, D.M. Goldberg, The red wine phenolics trans-resveratrol and quercetin block human platelet aggregation and eicosanoid synthesis: implications for protection against coronary heart disease, Clin. Chim. Acta 235 (1995) 207-219.

[9] Dorozynski, Wine may prevent dementia, BMJ 314 (1997) 993.

[10] A.I. Romero-Perez, M. Ibern-Gomez, R.M. Lamuela-Raventos, M.C. de la TorreBoronat, Piceid, the major resveratrol derivative in grape juices, J. Agric. Food Chem. 47 (1999) 1533-1536.

[11] G. Regev-Shoshani, O. Shoseyov, I. Bilkis, Z. Kerem, Glycosylation of resveratrol protects it from enzymic oxidation, Biochem. J. 374 (2003) 157-163.

12] G.J. Soleas, D.M. Goldberg, L. Grass, M. Levesque, E.P. Diamandis, Do wine polyphenols modulate p53 gene expression in human cancer cell lines? Clin. Biochem. 24 (2001) 415-420.

[13] M. Jang, L. Cai, G.O. Udeani, K.V. Slowing, C.F. Thomas, C.W. Beecher, H.H. Fong, N.R Farnsworth, A.D. Kinghorn, R.G. Mehta, R.C. Moon, J.M. Pezzuto, Cancer chemopreventive activity of resveratrol, a natural product derived from grapes, Science 275 (1997) 218-220.

[14] M.I. Chung, C.M. Teng, K.L. Cheng, F.N. Ko, C.N. Lin, An antiplatelet principle of Veratrum formosanum, Planta Med. 58 (1992) 274-276.

[15] F. Orsini, F. Pelizzoni, L. Verotta, T. Aburjai, C.B. Rogers, Isolation, synthesis, and antiplatelet aggregation activity of resveratrol 3-O-beta-D-glucopyranoside and related compounds, J. Nat. Prod. 60 (1997) 1082-1087.

[16] M.S. Pour Nikfardjam, G. Laszlo, H. Dietrich, Resveratrol-derivatives and antioxidative capacity in wines made from botrytized grapes, Food Chem. 96 (2006) 74-79.

[17] D. Huang, B. Ou, R.L. Prior, The chemistry behind antioxidant capacity assays, J. Agric. Food Chem. 53 (2005) 1841-1856.

[18] H. Mowri, S. Nojima, K. Inoue, Effect of lipid composition of liposomes on their sensitivity to peroxidation, J. Biochem. 95 (1984) 551-558.

[19] P. Viani, G. Cervato, A. Fiorilli, E. Rigomonti, B. Cestaio, Studies on peroxidation processes of model membranes and synaptosomes: role of phosphatidic acid, Chem. Phys. Lipids 52 (1990) 49-55.

[20] L.R. McLean, K.A. Hagaman, Effect of lipid physical state on the rate of peroxidation of liposomes, Free Radic. Biol. Med. 12 (1992) 113-119.

[21] G. Ravagnan, R. Falchetti, G. Lanzilli, M.P. Fuggetta, M. Tricarico and F. Mattivi, Use of extracts from spermatophyte plants with immunomodulating activity, Pat. EP 1 292320.

[22] F. Castelli, D. Trombetta, A. Tomaino, F. Bonina, G. Romeo, N. Uccella, A. Saija, Dipalmitoylphosphatidylcholine/linoleic acid mixed unilamellar vescicles as model membranes for studies on novel free-radical scavengers, J. Pharmacol. Toxicol. Methods 37 (1997) 135-141.

[23] Zhou, Q. Miao, L. Yang, Z.L. Liu, Antioxidative effects of flavonols and their glycosides against the free-radical-induced peroxidation of linoleic acid in solution and in micelles, Chem. Eur. J. 11 (2005) 680-691.

[24] M.S. Blois, antioxidant determinations by the use of a stable free radical, Nature 181 (1958) 1199-1200.

[25] Fauconneau, P. Waffo-Teguo, F. Huguet, L. Barrier, A. Decendit, J. Merillon, Comparative study of radical scavenger and antioxidant properties of phenolic compounds from Vitis vinifera cell cultures using in vitro tests, Life Sci. 61 (1997) 2103-2110.

[26] Kusumi, W.K. Subczynski, J.S. Hyde, Oxygen transport parameter in membranes as deduced by saturation recovery measurements of spin-lattice relaxation times of spin labels, Proc. Natl. Acad. Sci. U. S. A. 79 (1982) 1854-1858.

[27] L.D. Mayer, M.J. Hope, P.R. Cullis, Vescicles of variable sizes produced by a rapid extrusion procedure, Biochim. Biophys. Acta 858 (1986) 161-168.

[28] F. Momo, S. Fabris, R. Stevanato, Interaction of fluoxetine with phosphatidylcholine liposomes, Biophys. Chemist. 118 (2005) 15-21.

[29] J. Ancerewicz, E. Migliavacca, P.A. Carrupt, B. Testa, F. Bree, R. Zini, J.P. Tillement, S. Labidalle, D. Guyot, A.M. Chauvet-Monges, A. Crevet, A. Le Ridant, Structureproperty relationships of trimetazidine derivatives and model compounds as potential antioxidants, Free Radic. Biol. Med. 25 (1998) 113-120.

[30] W. Brand-Williams, M.E. Cuevelier, C. Berset, Use of a free radical method to evaluate antioxidant activity, Food Sci. Technol. 28 (1995) 25-30.

[31] J.S. Wright, E.R. Johnson, G.A. DiLabio, Predicting the activity of phenolic antioxidants: theoretical method, analysis of substituent effects, and application to major families of antioxidants, J. Am. Chem. Soc. 123 (2001) 1173-1183.

[32] W.A. Pryor, J.A. Cornicelli, L.J. Devall, B. Tait, B.K. Trivedi, D.T. Witiak, M. Wu, A rapid screening test to determine the antioxidant potencies of natural and synthetic antioxidants, J. Org. Chem. 58 (1993) 3521-3532.

[33] M.C. Hanlon, D.W. Seybert, The pH dependence of lipid peroxidation using watersoluble azo initiators, Free Radic. Biol. Med. 23 (1997) 712-719. 
[34] P. Langcake, R.J. Pryce, Oxidative dimerisation of 4-hydroxystilbenes in vitro: production of a grapevine phytoalexin mimic, J. Chem. Soc., Chem. Commun. (1977) 208-210.

[35] R. Petez, C. Perret, J.B. Jean-Denis, R. Tabacchi, K. Gindro, O. Viret, $\delta$-Viniferin, resveratrol dehydrodimer: one of the major stilbenes synthesized by stressed grapevine leaves, J. Agric. Food Chem. 51 (2003) 5488-5492.

[36] Thompson, K. Norbeck, L.I. Olsson, D. Costantin-Teodosium, J. Van der Zeeand, P. Moldeus, Peroxidase-catalyzed oxidation of eugenol: formation of a cytotoxic metabolite, J. Biol. Chem. 264 (1989) 1016-1021.

[37] S. Xu, G. Wang, H.M. Liu, L.J. Wang, H.F. Wang, A Dmol3 study on the reaction between trans-resveratrol and hydroperoxyl radical: dissimilarity of antioxidant activity among $\mathrm{O}-\mathrm{H}$ groups of trans-resveratrol, J. Mol. Struct., Theochem 809 (2007) 79-85.

[38] H. Cao, X. Pan, C. Li, C. Zhou, F. Deng, T. Li, Density functional theory calculations for resveratrol, Bioorg. Med. Chem. Lett. 13 (2003) 1869-1871.
[39] Marsch, in: E. Grell (Ed.), Electron Spin Resonance: Spin Labels, Membrane Spectroscopy, Springer-Verlag, New York, 1981, pp. 51-142.

[40] F. Momo, S. Fabris, A. Wisniewska, C. Fiore, A. Bindoli, G. Scutari, R. Stevanato, Correlation between fluidising effects on phospholipid membranes and mitochondrial respiration of propofol and p-nitrosophenols homologues, Biophys. Chemist. 103 (2003) 213-222.

[41] G.R. Buettner, The pecking order of free radicals and antioxidants: lipid peroxidation, $\alpha$-tocopherol, and ascorbate, Arch. Biochem. Biophys. 300 (1993) 535-543.

[42] L.R.C. Barclay, K.U. Ingold, Autoxidation of biological molecules. 2. Autoxidation of a model membrane. Comparison of the autoxidation of egg lecithin phosphatidylcholine in water and in chlorobenzene, J. Am. Chem. Soc. 103 (1981) 6478-6485. 